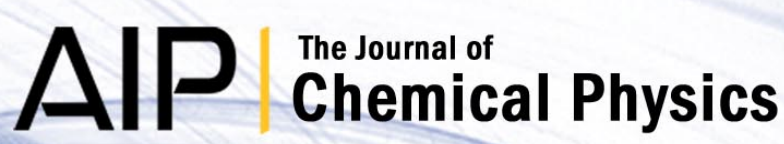

Adsorption of semiflexible block copolymers on homogeneous surfaces

J. J. Cerdà, T. Sintes, and K. Sumithra

Citation: J. Chem. Phys. 123, 204703 (2005); doi: 10.1063/1.2101526

View online: http://dx.doi.org/10.1063/1.2101526

View Table of Contents: http://jcp.aip.org/resource/1/JCPSA6/v123/i20

Published by the American Institute of Physics.

Additional information on J. Chem. Phys.

Journal Homepage: http://jcp.aip.org/

Journal Information: http://jcp.aip.org/about/about_the_journal

Top downloads: http://jcp.aip.org/features/most_downloaded

Information for Authors: http://jcp.aip.org/authors

Article-level metrics now available

Join the conversation! Rate $\&$ comment on articles 


\title{
Adsorption of semiflexible block copolymers on homogeneous surfaces
}

\author{
J. J. Cerdà and T. Sintes ${ }^{a)}$ \\ Departament de Física, Universitat de les Illes Balears, E-07122 Palma de Mallorca, Spain \\ K. Sumithra \\ FG Theoretische Physik, FB Physik, Martin Luther Universitaet, D-06099 Halle-Wittenberg, Germany
}

(Received 29 April 2005; accepted 6 September 2005; published online 18 November 2005)

\begin{abstract}
We present the results of extensive numerical off-lattice Monte Carlo simulations of semiflexible block-copolymer chains adsorbed onto flat homogeneous surfaces. We have compared the behavior of several chain structures, such as homopolymers, diblocks, $\left(A_{\alpha} B_{\alpha}\right)$ block copolymers, and random heteropolymers. In all the cases studied, we have found the adsorption process to be favored with an increase of the chain rigidity. Particularly, the adsorption of diblock structures becomes a two-step process characterized by two different adsorbing temperatures that depend on the chain stiffness $\kappa$, the chain length $N$, and the adsorbing energies $\epsilon_{A}$ and $\epsilon_{B}$. This twofold adsorbing process changes to a single one for copolymers of reduced block size $\alpha$. Each block of the stiff copolymer chain is found to satisfy the classical scaling laws for flexible chains, however, we found the scaling exponent $\phi$ to depend on the chain stiffness. The measurement of the radius of gyration exhibits a typical behavior of a polymer chain composed of $N / l_{p}$ blobs whose persistence length follows $l_{p} \sim\left(\kappa / k_{B} T\right)^{0.5}$ for large stiff chains. (C) 2005 American Institute of Physics.

[DOI: $10.1063 / 1.2101526]$
\end{abstract}

\section{INTRODUCTION}

Polymer adsorption has been a topic of great experimental and theoretical interests due to its enormous number of applications in science and technology. These applications cover the stabilization of colloidal suspensions, adhesion, gel permeation, chromatography, etc. It is a characteristic feature of all these systems that their physical properties are mediated by the interaction between polymer molecules and an impenetrable surface. The bulk conformational properties of these polymer chains are strongly modified in contact with the surface due to a subtle competition between the loss of entropy at the surface and the gain of internal energy.

In the last decade, a large number of theoretical studies have been devoted to the adsorption of flexible homopolymers, ${ }^{1-7}$ diblock copolymers, ${ }^{8-10} A B$-type alternating copolymers, ${ }^{11-13}$ and random copolymers. ${ }^{14-16,19}$ In turn, the adsorbing surface has been considered as flat and homogeneous $^{1-5,8-16}$ or heterogeneous, ${ }^{6,7,17,18}$ including regions with a random distribution of adsorption energies.

However, many polymers are rigid up to a certain extent due to steric and electronic delocalization effects. Few important examples of such stiff chains are found in synthetic macromolecules and specially in biology, just to mention, DNA, collagen, microtubules, and actin filaments. ${ }^{19}$ On a local length scale, the stiffness is very prominent and it is expected to produce significant changes in the macromolecular properties. Among the many significant phenomena induced by the polymer stiffness we can mention the nematic liquid-crystalline ordering of semiflexible polymer segments.

The constrained rotational motions of the monomers

${ }^{a)}$ Electronic mail: tomas@imedea.uib.es about the chain backbone make the use of conventional models developed for flexible chains inadequate. The statistical mechanics of such chains was first formulated by Kratky and Porod, ${ }^{20}$ about 50 years ago, and it is still considered as a nontrivial problem. In contraposition to flexible chains, the number of studies devoted to stiff polymer chains is scarce and focused on the behavior of homopolymer structures. ${ }^{21-25}$

Copolymer adsorption onto surfaces has become recently a topic of great interest in the design of novel bloodcontacting materials for medical implants and bioaffinity sensors. ${ }^{26,27}$ The adsorption of copolymeric chains has also found applications in nanotechnology. For instance, pluronic triblock copolymers are used in order to activate external wall carbon nanotubes and, at the same time, to prevent nonspecific bounding of molecules onto nanotube surfaces. ${ }^{28}$ Such coated nanotubes are the basis for the design of highly specific electronic biosensors. Furthermore, copolymer absorption plays an important role in the design of dynamic polymeric wall coatings for capillary electrophoresis. ${ }^{29}$ Polymer properties help to create an ideal wall coating with a notorious impact on the electro-osmotic flow capabilities.

The purpose of the present paper is to study how the adsorption properties of stiff polymers are modified when the chain structure is changed and to examine whether the universal scaling laws apply in these systems. We will present the results of extensive off-lattice Monte Carlo simulations of semiflexible block-copolymer chains adsorbing onto flat homogeneous surfaces. The polymer will contain two types of monomers $A$ and $B$ forming blocks of different sizes that interact with the surface with energies $\epsilon_{A}$ and $\epsilon_{B}$, respectively. Besides the block size, we will characterize the adsorption process as a function of the chain rigidity and the chain length. Our simulations are single chain. Although ap- 
plications usually involve many interacting chains at the surface, the adsorption of isolated chains provides a valuable benchmark for studies of many-chain systems and is directly relevant to a number of emerging single-molecule approaches to molecular genomics. ${ }^{30,31}$

The rest of the paper is organized as follows: in Sec. II we describe the numerical model, in Sec. III we present the results for diblock semiflexible chains and block-copolymer structures, and Sec. IV concludes with a brief summary and a discussion of the results.

\section{NUMERICAL MODEL}

We have studied the adsorption of a semiflexible blockcopolymer chain onto a homogeneous surface using offlattice Monte Carlo simulations. We have considered the polymer chain to be confined in a unit box with periodic boundary conditions in the $x-y$ domain. The length of the cell is chosen to be at least twice the chain length $N$, and an impenetrable planar surface is set at the plane $z=0$. The polymer chain is represented by a pearl-necklace model ${ }^{32}$ containing $N$ beads of diameter $\sigma$.

The stiffness of the polymer chain is usually characterized by the arclength over which the tangential correlations of the contour decay to $1 / e$ and it is commonly referred as the persistence length $l_{p}$. In other words, it can be considered as the distance over which the polymer chain is stiff. We introduce the stiffness through the bending potential $U_{\text {ben }}$ given by ${ }^{33}$

$$
U_{\text {bend }}=\sum_{\theta} \kappa(1+\cos \theta)^{2}
$$

where $\theta$ is the bond angle between any three consecutive beads and $\kappa$ is the bending constant.

Monomers that are two or more beads apart interact through a steric hard-core potential of the form

$$
U_{\text {steric }}=\sum_{i, j=1}^{N} V\left(r_{i j}\right) \text {, }
$$

where $V$ is given by

$$
V\left(r_{i j}\right)= \begin{cases}0 & \text { for }\left|\mathbf{r}_{i}-\mathbf{r}_{j}\right|>\sigma \\ \infty & \text { for }\left|\mathbf{r}_{i}-\mathbf{r}_{j}\right|<\sigma .\end{cases}
$$

Monomer units can be of type $A$ or $B$ and are distributed along the chain of length $N$ according to the selected polymer structure. A diblock copolymer will be denoted by $\left(A_{N / 2} B_{N / 2}\right)$ and, in general, a block copolymer formed by $\alpha$ monomers of type $A$ followed by $\alpha$ monomers of type $B$, repeating this sequence along the entire chain, by $\left(A_{\alpha} B_{\alpha}\right)_{N / 2 \alpha}$.

Monomers of types $A$ and $B$ interact with the impenetrable surface at $z=0$ with adsorption energies $\epsilon_{A}$ and $\epsilon_{B}$, respectively. Thus, we define an adsorption potential $U_{\text {ads }}$ as

$$
U_{\mathrm{ads}}=n_{A} \epsilon_{A}+n_{B} \epsilon_{B},
$$

where $n_{A}$ and $n_{B}$ are the numbers of $A$-type and $B$-type monomers such that their $z$ coordinate verifies $0<z<\sigma$. The total energy of the system will contain the above contributions and is written as

$$
U=U_{\text {ads }}+U_{\text {bend }}+U_{\text {steric }} \text {. }
$$

The initial configuration of the self-avoiding polymer is randomly generated with one monomer attached to the surface $z=0$. Next, the polymer tried to move by randomly executing a repeating-type motion in a forward or reverse direction, or by selecting an individual monomer which position can be rotated around the axis connecting the previous and following monomers in the chain in an arbitrary angle between 0 and $2 \pi$. Chain ends just perform random wiggling motions. Each move is accepted according to the standard Metropolis Monte Carlo algorithm: $\exp \left(-\Delta U / k_{B} T\right)>\eta$, where $0<\eta<1$ is a flat random number. A link-cell method $^{34}$ has been implemented in the algorithm to efficiently check $\Delta U$.

At very high temperatures, or equivalently at low adsorption energies, the chain has a tendency to diffuse into the bulk. We prevent this by forcing the polymer to have at least one monomer attached to the surface. However, one does not expect any significant change in the statistical properties of the adsorbed chain at low temperatures. We define one Monte Carlo step (MCS) as $N$ trials to move the chain. The system has been equilibrated for $5 \times 10^{5}$ MCSs. Subsequently, the number of monomers in contact with the surface is evaluated every ten MCSs. The results have been finally averaged over $10^{6}$ measures. In the case of random $A B$ copolymers, additional averages over ten different sequences have been performed. For the rest of the paper we have taken $\sigma=1.0$. The temperature is given in units of $1 / k_{B}$, hence $T \equiv k_{B} T$.

\section{RESULTS AND DISCUSSION}

\section{A. Diblock chains}

In this section we will focus in the study of the behavior of stiff diblock-polymer chains adsorbing onto a planar surface. A diblock chain of length $N$ consists in two segments of equal size $N / 2$, the first one composed of monomers of type $A$, followed by a second block containing monomers of type $B$. Monomers $A$ and $B$ interact with the surface with energies $\epsilon_{A}$ and $\epsilon_{B}$, respectively. A negative adsorbing energy stands for an attractive interaction whereas a positive value implies repulsion. Through the simulations we have set $\epsilon_{A}=-1$ and we have studied the transition towards the adsorbed state as a function of the chain length $N$, the stiffness parameter $\kappa$, and the interaction energy $\epsilon_{B}$.

In Fig. 1 we have plotted the change in the absolute value of the averaged adsorbing energy per monomer $E / N$ $=\left\langle n_{A} \epsilon_{A}+n_{B} \epsilon_{B}\right\rangle / N$ as a function of the inverse of the temperature $1 / T$, for different values of the stiffness $\kappa$ and for a fixed adsorbing energy $\epsilon_{B}=-0.5$. Figure 1 (a) stands for short polymer chains $(N=20)$, while Fig. $1(\mathrm{~b})$ is devoted to long chains $(N=300)$.

The behavior observed for stiff diblocks is very similar to the one found for semiflexible homopolymers. ${ }^{22,23}$ Stiffer chains adsorb more easily onto the surface with increasing $\kappa$ and, therefore, they are characterized by a higher critical adsorption temperature $T_{c}$. The limiting values observed for $T \rightarrow \infty$ correspond to a chain completely adsorbed onto the 

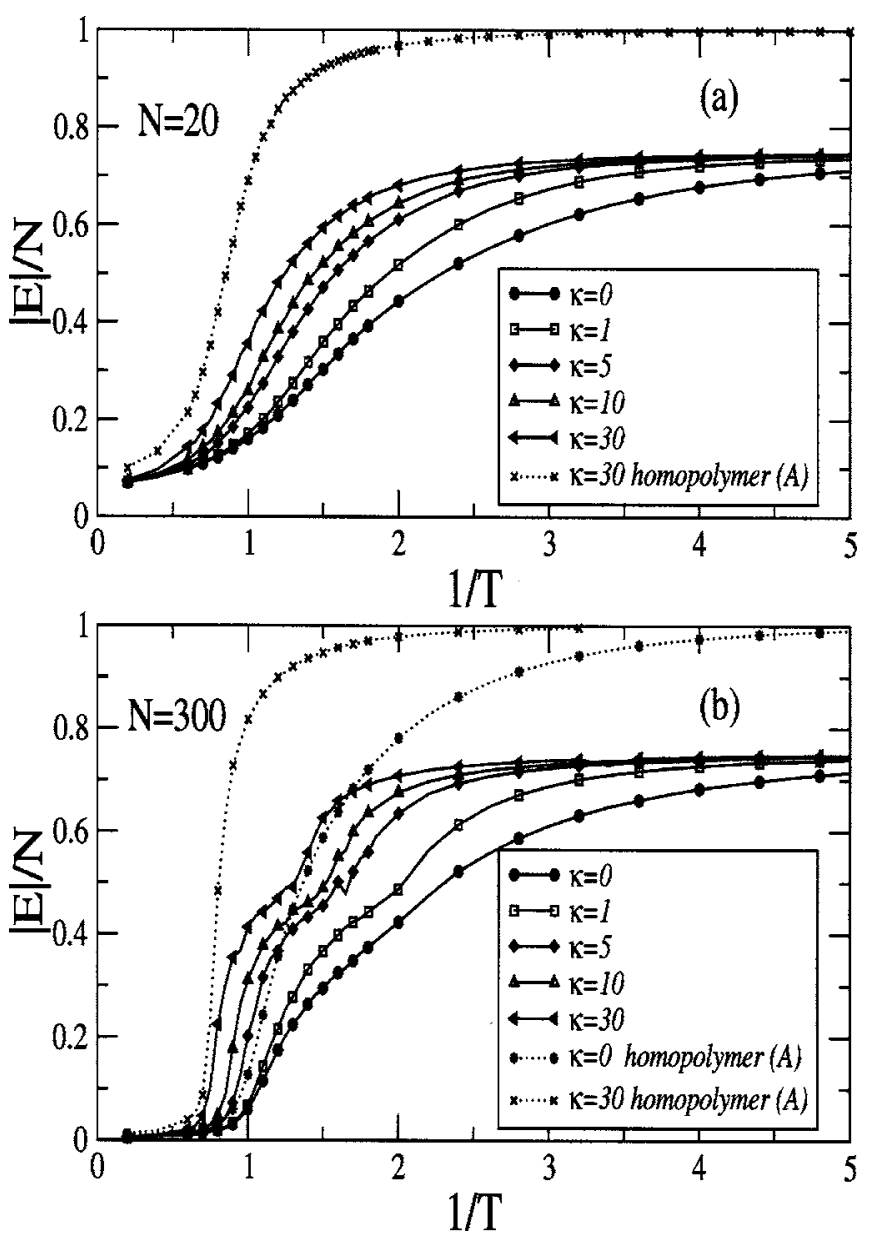

FIG. 1. Averaged adsorption energy per monomer in diblock chains vs the inverse of the temperature for different values of the stiffness parameter $\kappa$. The interaction energies are set to $\epsilon_{A}=-1$ and $\epsilon_{B}=-0.5$. (a) For a short chain $N=20$ and (b) for a long chain $N=300$. For comparison we include the results for a homopolymer composed of monomers of type $A$ for flexible $(\kappa=0)$ and stiff chains $(\kappa=30)$. Note how for increasing the chain length the adsorption becomes a two-step process.

surface with $|E| / N=1 / 2\left|\epsilon_{A}+\epsilon_{B}\right|$. A remarkable difference with respect to the homopolymer adsorption becomes notorious for increasing chain lengths: the adsorption process becomes a two-step process. Since $A$-type monomers interact more strongly with the surface than $B$-type monomers, $\left|\epsilon_{A}\right|$ $>\left|\epsilon_{B}\right|$, block $A$ adsorbs onto the surface at a higher characteristic temperature than block $B, T_{c A}>T_{c B}$, as can be seen in Fig. 1(b). These critical adsorbing temperatures are determined by the position of the maxima of the specific-heat data, as described below. For comparison, we have also included in Fig. 1(b) the averaged adsorption energy of a homopolymer of equal length, composed with $A$-type monomers only, and with rigidities $\kappa=0$ (flexible) and $\kappa=30$. We can observe that for stiff chains, the characteristic temperature at which the transition to the adsorbed state takes place for the homopolymer (A-type) is very similar to the one found for the block $A$ in the diblock chain.

The effect of changing $\epsilon_{B}$ on the adsorption process is shown in Fig. 2. The rest of parameters are set to $N=200$ and $\kappa=30$. For an attractive interaction $\left(\epsilon_{B}<0\right)$ the block $B$ adsorbs onto the surface at a characteristic temperature that increases with the intensity of the interaction, as expected.

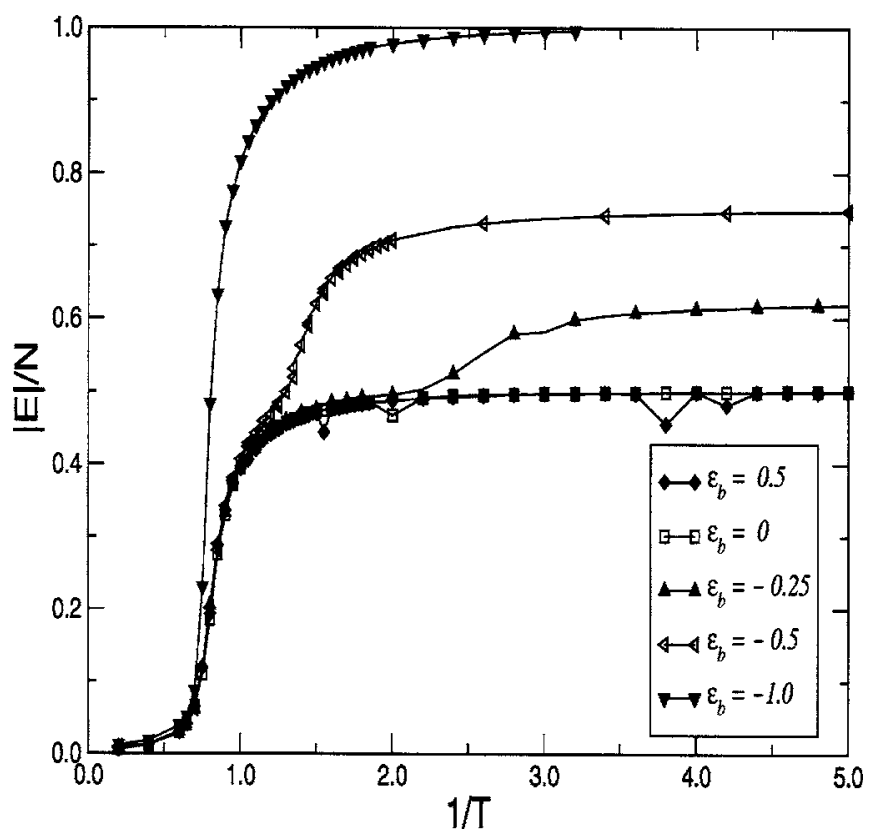

FIG. 2. Averaged adsorption energy per monomer vs the inverse of the temperature for different values of $\epsilon_{B}$. The other parameters are set to $N=200, \kappa=30$, and $\epsilon_{A}=-1$. Note how the adsorption process becomes independent of $\epsilon_{B}$ for $\epsilon_{B} \geqslant 0$.

On the other hand, for $\epsilon_{B} \geqslant 0$ the block $B$ does not adsorb at all. Since the repulsion is short ranged, $\epsilon_{B}$ has no effect on the adsorption process and the system is dominated purely by entropic forces.

The characteristic adsorption temperatures at which the chain adsorbs onto the surface can be determined from the position of the maximum in the specific heat that is obtained by calculating the fluctuations of the energy. The results for $N=150$ and $\kappa=30$, for different values of $\epsilon_{B}$, are presented in Fig. 3. A single peak appears for $\epsilon_{B}>0$ corresponding to the characteristic adsorption temperature of block $A$ alone. For

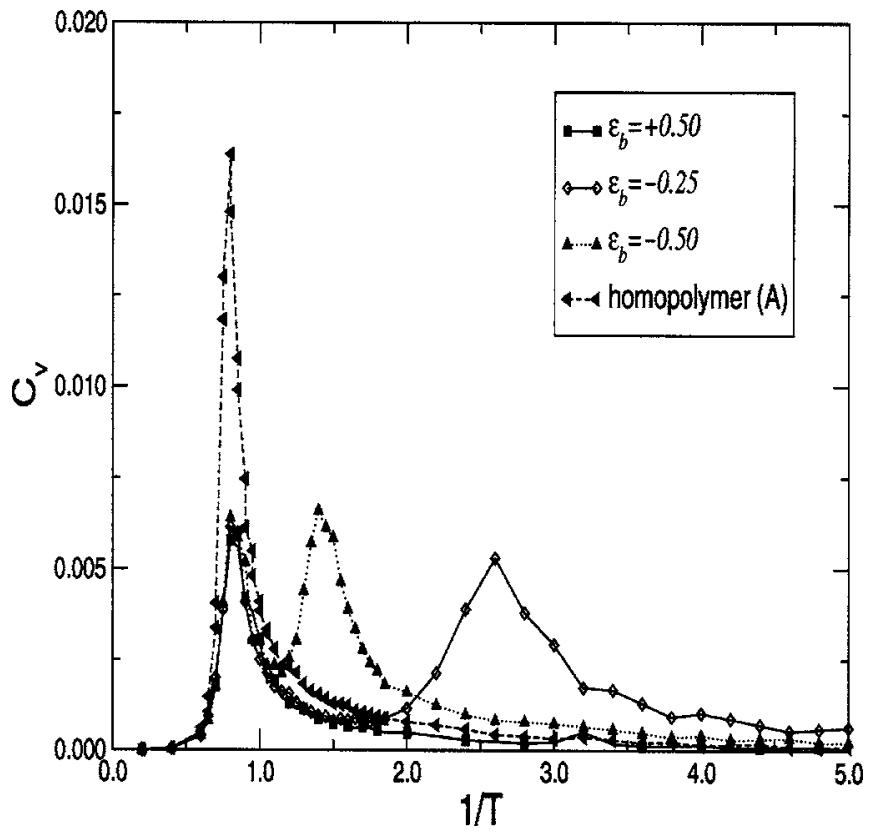

FIG. 3. Specific heat of a stiff diblock chain vs $1 / T$ for different adsorption energies $\epsilon_{B}$. The other parameters are set to $N=150, \kappa=30$, and $\epsilon_{A}=-1$. 


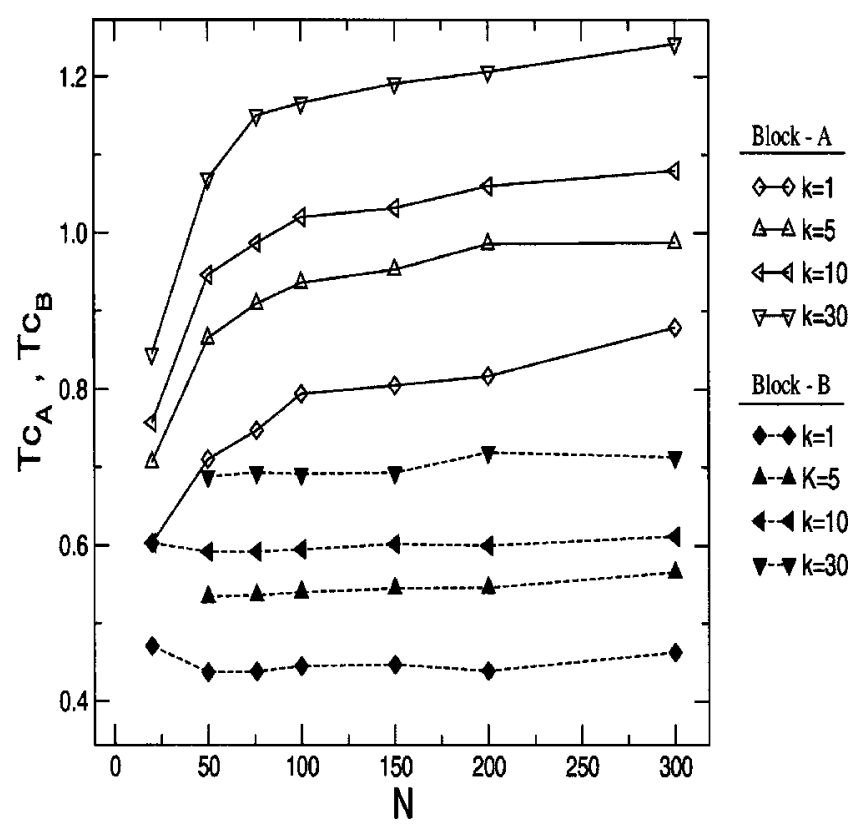

FIG. 4. Transition temperatures for the block- $A T_{c A}\left(N, \kappa, \epsilon_{A}\right)$ (opened symbols) and for the block- $B T_{c B}\left(N, \kappa, \epsilon_{B}\right)$ (filled symbols) vs the chain length N. $\epsilon_{A}=-1$ and $\epsilon_{B}=-0.5$.

an attractive interaction we can observe two maxima related to $T_{c A}$ and $T_{c B}$. It is also clear that the adsorption transition becomes sharper as $\left|\epsilon_{B}\right|$ increases. We should remark that at a constant chain length and stiffness, the characteristic adsorption temperature for the block $A$ is practically unaffected by tuning $\epsilon_{B}$. To make clear the dependence of the transition temperature with the chain stiffness and the intensity of the interaction with the surface, we have plotted in Fig. 4 $T_{c}(N, \kappa, \epsilon)$ as a function of the chain length $N$. The characteristic adsorption temperature is found to increase with the chain length and rigidity, as expected, and specially with the interaction with the surface, where the differences between $T_{c A}$ for $\epsilon_{A}=-1$ and $T_{c B}$ with $\epsilon_{B}=-0.5$ are remarkable even for the same stiffness $\kappa$.

In order to examine the critical adsorption properties of semiflexible block copolymers, we will assume initially the scaling ansatz proposed by Kramarenko et al. for flexible homopolymers. ${ }^{23}$ We have determined the critical adsorption temperature for $N \rightarrow \infty, T_{c}(\infty, \kappa, \epsilon)$, and using these values we have done a scaling analysis of the data for the fraction of adsorbed monomers $\chi \equiv n / N$ as a function of the scaling variable $\tau N^{\phi} . n$ is the number of adsorbed monomers, $N$ is the chain length, and $\tau=\left(T-T_{c}(\infty, \kappa, \epsilon)\right) / T_{c}(\infty, \kappa, \epsilon)$ is the temperature distance to the critical temperature. For a given value of the stiffness and the interaction energy, the crossover scaling for the fraction of adsorbed monomers can be expressed as

$$
\chi / N^{\phi-1}=h\left(\tau N^{\phi}\right) .
$$

In order to test our Monte Carlo method, we have derived first the results for flexible homopolymers. In this case, we obtained an exponent $\phi=0.59 \pm 0.02$ in agreement with the well-known scaling exponent-found by Eisenriegler et al. ${ }^{35}$ and de Gennes and Pincus, ${ }^{36}$ also verified by Kramarenko et $a l .{ }^{23}$ This results is shown in the inset plot of Fig. 5. How-

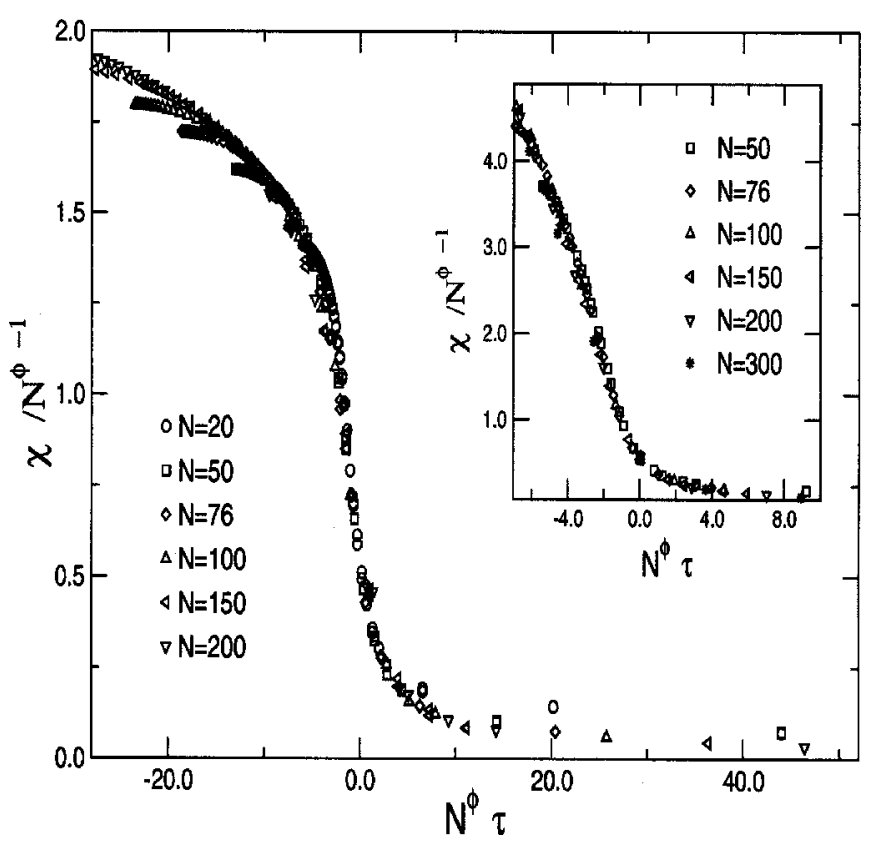

FIG. 5. Scaling function $\chi / N^{\phi-1}$ vs the scaling argument $\tau N^{\phi}$ for a semiflexible homopolymer with an adsorption energy $\epsilon=-1$ and a chain stiffness $\kappa=30$. The best scaling exponent found is $\phi=0.85$. Inset plot: the same scaling function for flexible homopolymers $(\kappa=0)$ with $\phi=0.59$.

ever, for homopolymers with a large degree of stiffness we were unable to find the same scaling exponent. The best scaling exponent we have found for $\kappa=30$ is $\phi=0.85 \pm 0.05$ (Fig. 5), far from the expected value for flexible chains. The fact that there can be a range of values of $\phi$ that do not change appreciably the scaling has been observed in the paper of Moghaddam et al. ${ }^{12}$ studying the behavior of a self avoiding walk (SAW) copolymer onto a cubic lattice, thus, for small values of $\kappa$ one could even scale the data with $\phi \approx 0.59$ with no appreciable differences. ${ }^{22}$ The situation changes completely for large $\kappa$. In this sense, Kuznetsov and Sung $^{5}$ showed that the deadsorbed-adsorbed transition for semiflexible polymers is an outcome of the interplay between the shape of the surface attraction, including the thickness of the adsorption layer, the thermal fluctuations, and the chain stiffness. This nonuniversal scaling behavior has been also evidenced in the work of Gompper, ${ }^{37}$ where three distinct subregimes were expected due to the dependence of the scaling exponent $\phi$ with the potential shape acting on the monomers. Thus, a good scaling relation for semiflexible polymer chains must be a function of stiffness parameter $\kappa$ and the adsorption energy $\epsilon$. In general, in our simulations we observe that as the degree of stiffness increases, the best value for the critical exponent $\phi$ in order scale the data also increases.

In Fig. 6 we show the scaling of stiff diblock chains $(\kappa=30)$ with $\epsilon_{A}=-1$ and $\epsilon_{B}=-0.5$. In order to check whether the presence of blocks with different adsorbing energies might affect the value of the crossover scaling exponent, we have scaled, separately, each block introducing two different exponents, $\phi_{A}$ and $\phi_{B}$, corresponding to the blocks with adsorbing energies $\epsilon_{A}$ and $\epsilon_{B}$, respectively. We have found $\phi_{A}=\phi_{B}=0.80 \pm 0.05$. Only deviations are found for short polymer chains. These deviations could be originated in the 


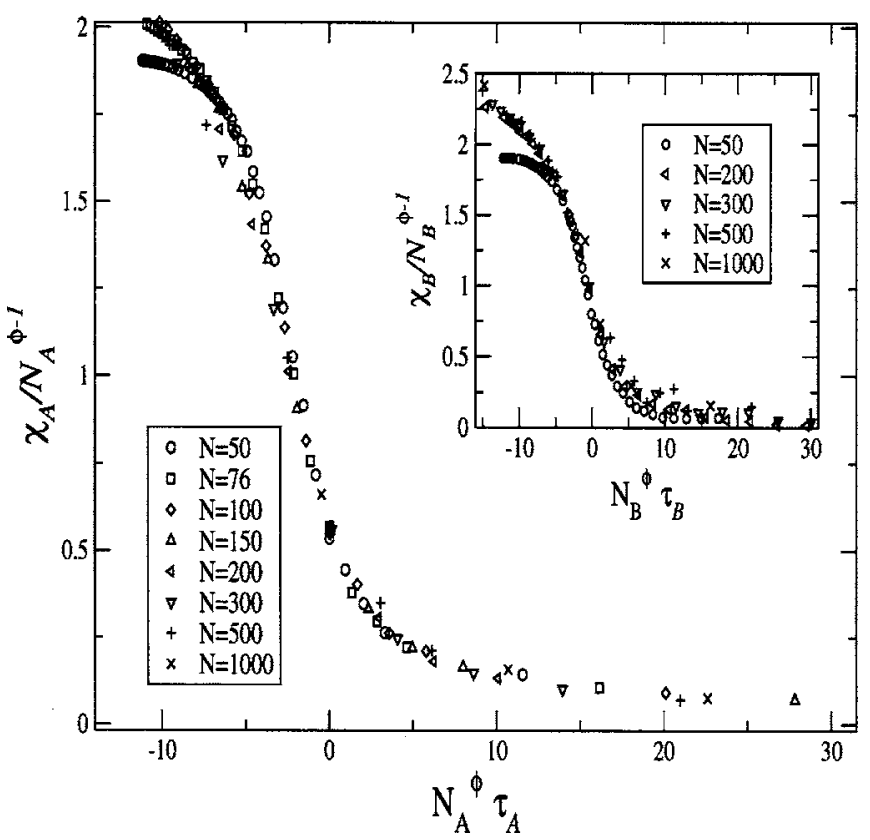

FIG. 6. Scaling function $\chi / N^{\phi-1}$ vs the scaling variable $\tau N^{\phi}$ for block $A$ of a semiflexible diblock chain with adsorption energies $\epsilon_{A}=-1$ and $\epsilon_{B}=-0.5$. The chain stiffness is set to $\kappa=30$. Inset plot: same as before for the block $B$. Blocks $A$ and $B$ are found to satisfy the same scaling exponent $\phi_{A}=\phi_{B}$ $=0.8$, similar to the one found for semiflexible homopolymers with the same rigidity.

perturbations near the junction point between the $A$ and $B$ blocks. In fact, we had to simulate larger polymer chains (up to $N=1000$ ) to recover a result that is in agreement with the one found for a homopolymer of the same rigidity, supposedly to make such perturbations negligible.

In comparison with our results, a recent study of Whittington $^{11}$ deduced analytically for a lattice-flexible SAW, in the limit $N \rightarrow \infty$, that a homopolymer of $N_{A}$ monomers has the same critical adsorbing temperature $T_{C}$ than a block copolymer of $N_{A}$ monomers in which the $B$-type monomers interact with the surface with an energy $\epsilon_{B}=0$. This result is in agreement with the outcome of Fig. 2. Moreover, the value of $T_{c A}$ is practically unaffected by the presence of the block $B$ in any case, and it is very close to the corresponding $T_{c}$ for a homopolymer composed of $A$-type monomers (see Fig. 3). As a result, Whittington expected a same value of $\phi$ for these chain structures, only $A B$-alternating chains were an exception. This result is also in agreement with our findings (Fig. 6). Thus, our numerical results suggest that the findings of Whittington, derived for flexible chains, could be extended to stiff polymer chains, with a scaling exponent that depends on the interaction parameters, $\phi(\kappa)$, even in the case where $\epsilon_{B} \neq 0$.

In order to characterize the behavior of semiflexible polymer chains, another magnitude of interest is the radius of gyration. In Fig. 7 we show the components of the radius of gyration parallel and perpendicular to the adsorbing surface for chains with different rigidities. The adsorbing energies have been selected to be $\epsilon_{A}=-1$ and $\epsilon_{B}=-0.5$. It is clear from the picture that with increasing $\kappa$, the parallel component reaches to its maximum value and, accordingly, the perpendicular component drops to zero (the polymer is fully ad-

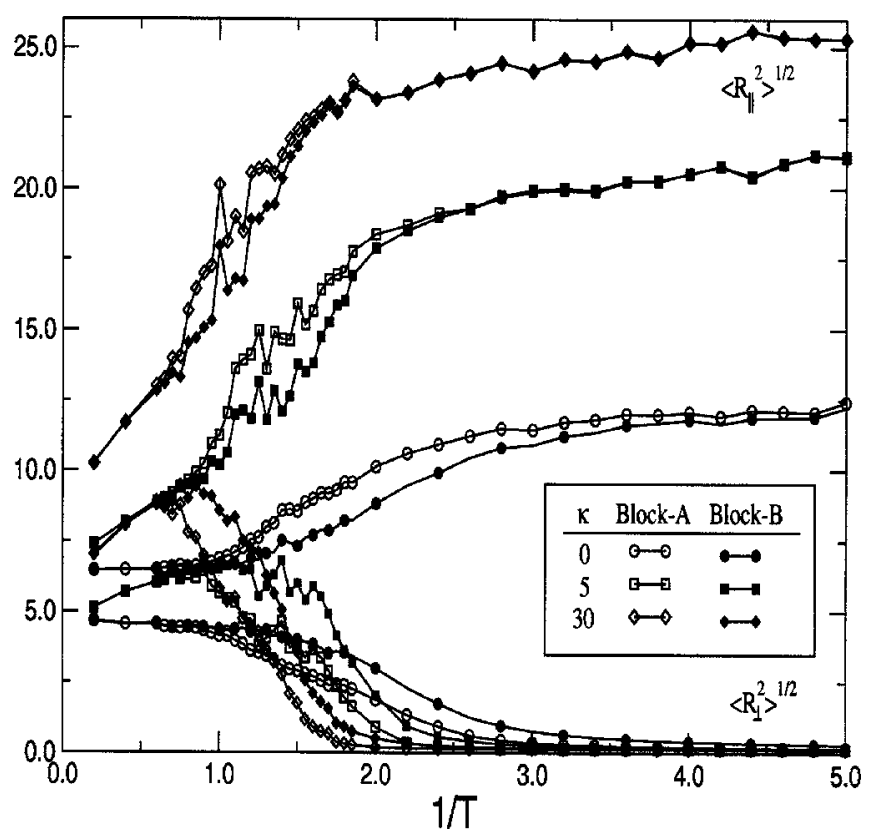

FIG. 7. Parallel and perpendicular radius of gyration for the $A$ block (open symbols) and the $B$ block (filled symbols) of a diblock-polymer chain for several degrees of stiffness $\kappa$. The chain length is set to $N=100 . \epsilon_{A}=-1$ and $\epsilon_{B}=-0.5$.

sorbed), at earlier times, that is, at higher temperatures. The two different blocks also display a different behavior due to the different adsorbing energies. The higher $|\epsilon|$ the higher is the temperature at which the block adsorbs. In Fig. 8 we show the effect of $\epsilon_{B}$ on the components of the radius of gyration associated with the block $A$ for a fixed chain stiffness. The less adsorbing block $B$ hampers the block $A$ to be completely adsorbed at its characteristic adsorbing temperature. A complete adsorption of the block $A$ only takes place when the whole chain is adsorbed.

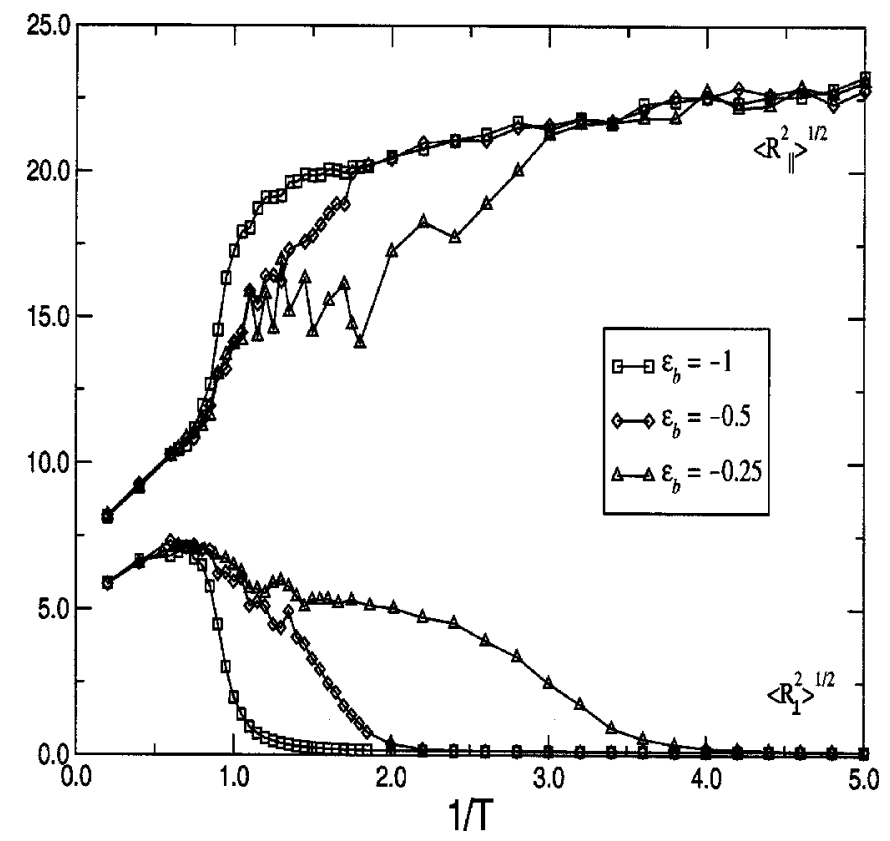

FIG. 8. Comparison between the parallel and perpendicular radius of gyration for the $A$ block for different adsorbtion energies for the $B$-type monomers $\epsilon_{B}$. Diblock chains have stiffness $\kappa=10$, chain length $N=100$, and $\epsilon_{A}=-1$. 


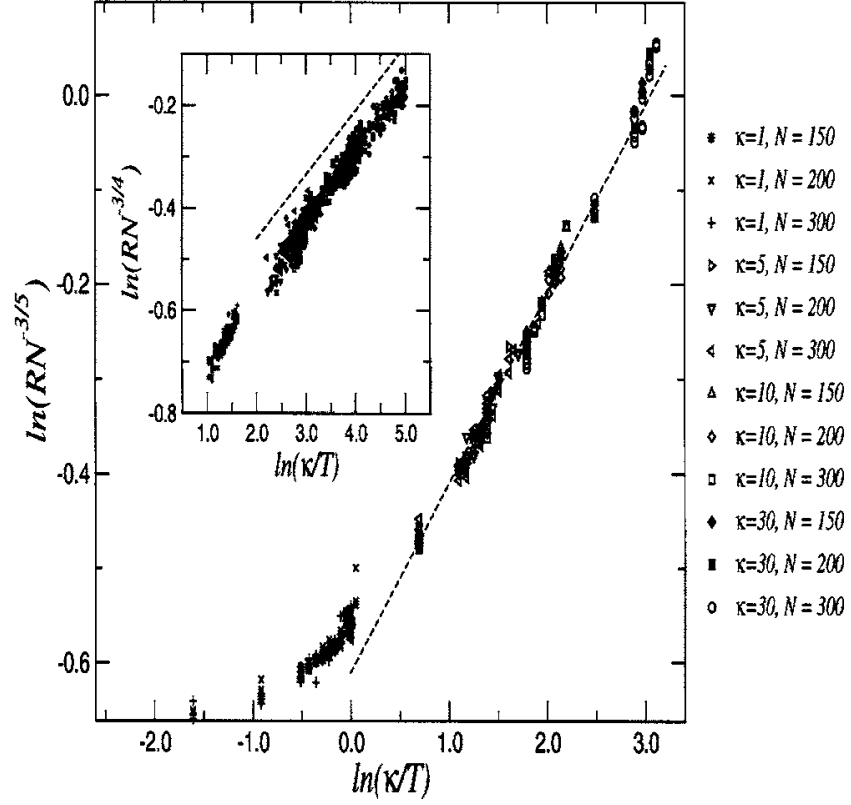

FIG. 9. Scaling plot for semiflexible diblock chains in the unadsorbed state. $\kappa=10, \epsilon_{A}=-1$, and $\epsilon_{B}=-0.5$. A dashed line of slope 0.2 is included to guide the eye. Inset: same scaling plot in the adsorbed state (the system is now two dimensional). A dashed line of slope 0.125 is included to guide the eye.

The behavior of the radius of gyration with the chain stiffness can be easily explained. Assume that a semiflexible diblock chain has a persistence length given by

$$
l_{p} \sim\left(\frac{\kappa}{T}\right)^{\alpha}
$$

where $\alpha \rightarrow 0.5$ in the limit $\kappa / T \rightarrow \infty{ }^{22}$ Thus, we can model the chain as an ideal rodlike polymer of $N / l_{p}$ units which the radius of gyration is proportional to

$$
R \sim l_{p}\left(\frac{N}{l_{p}}\right)^{\nu} \sim N^{\nu}\left(\frac{\kappa}{T}\right)^{\alpha(1-\nu)},
$$

where $\nu=3 /(d+2)$ is the Flory exponent that depends on the space dimensionality $d$. In Fig. 9 we analyze the scaling of $R N^{-\nu}$ vs $\kappa / T$. For semiflexible diblock chains in the unadsorbed state, a slope of $\alpha(1-\nu)=0.2$ is expected; whereas in the adsorbed state (inset of Fig. 9), the system becomes twodimensional and $\alpha(1-\nu)=0.125$. Both regimes have been confirmed by our numerical data.

\section{B. Block-copolymer chains}

In this section we shall study the adsorption process of block copolymers formed by a repeated sequence of $N /(2 \alpha)$ units $A_{\alpha} B_{\alpha}$. In Fig. 10 we have plotted the averaged adsorbed energy per monomer for different stiff copolymer structures generated by changing the value of $\alpha$. The rest of the parameters for this figure are fixed to $\kappa=10, N=200, \epsilon_{A}=-1$, and $\epsilon_{B}=-0.5$. In order to compare with previous results, the diblock structure and a homopolymer formed by $A$-type monomers are also included. As in the diblock case, $|E| / N$ $\rightarrow 1 / 2\left|\epsilon_{A}+\epsilon_{B}\right|$ in the limit $1 / T \rightarrow \infty$, corresponding to a fully adsorbed chain. It is remarkable to note that the two-step adsorbing process observed in the diblock case, that deter-

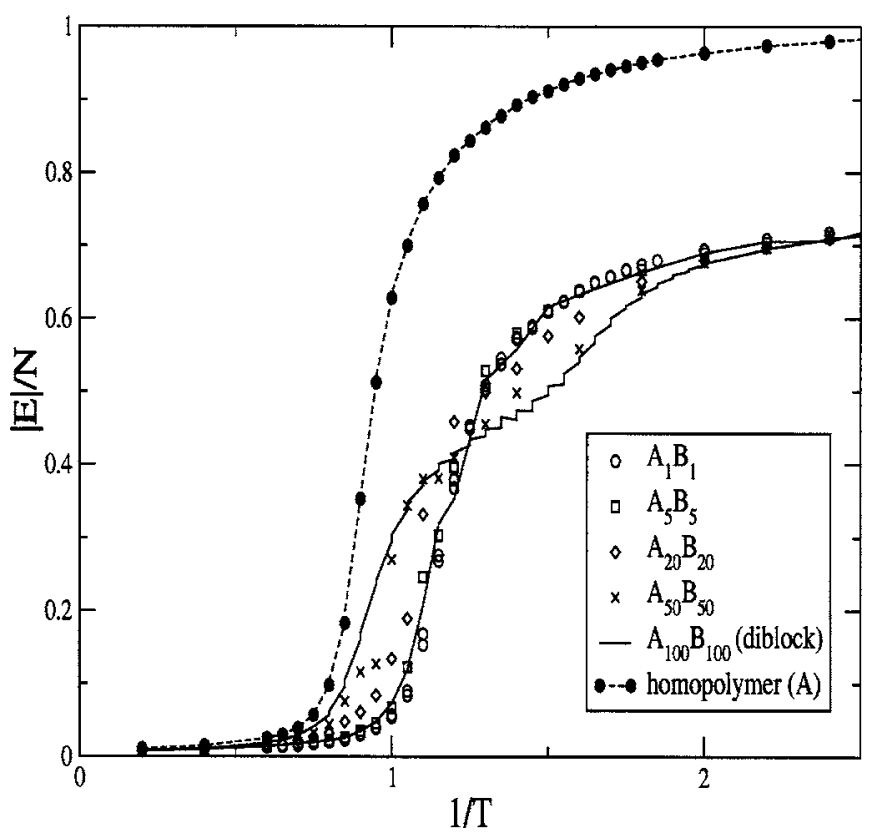

FIG. 10. Averaged adsorption energy per monomer for several blockcopolymer structures vs $1 / T$. The rest of the parameters are set to $\kappa=10$, $N=200, \epsilon_{A}=-1$, and $\epsilon_{B}=-0.5$. For comparison an $A$-type homopolymer is also included.

mines the existence to two characteristic adsorbing temperatures $T_{c A}$ and $T_{c B}$, vanishes for small values of $\alpha$. As a result, a single peak in the specific-heat data is expected.

The corresponding specific heat for the same polymer structures analyzed in Fig. 10 is shown in Fig. 11. For $A_{100} B_{100}$ (diblock) we can clearly observe two peaks whose location identifies the characteristic adsorbing temperatures

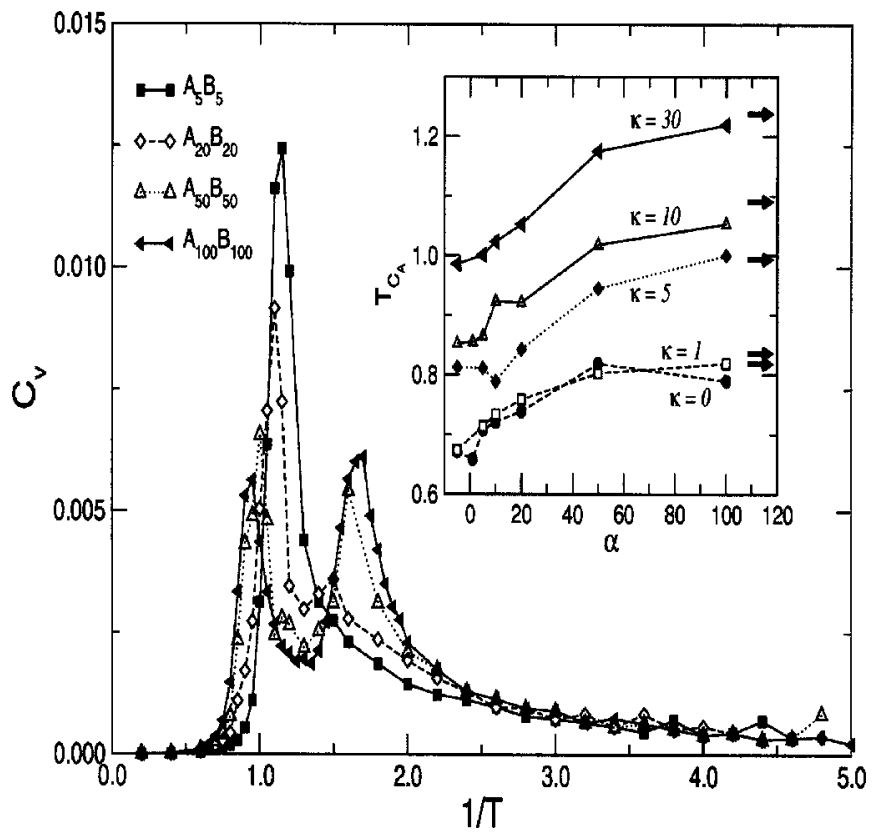

FIG. 11. Specific heat vs $1 / T$ for different copolymer structures with $\kappa=10, N=200, \epsilon_{A}=-1$, and $\epsilon_{B}=-0.5$. Inset: characteristic adsorption temperatures for the $A$ block, $T_{c A}$, vs the block size $\alpha$ for different chain rigidities $\kappa$. We have assigned, arbitrarily, $\alpha=-5$ to random $A B$ copolymers. The arrows indicate the value of $T_{c A}$ for an $A$-type homopolymer of the same length and rigidity. 


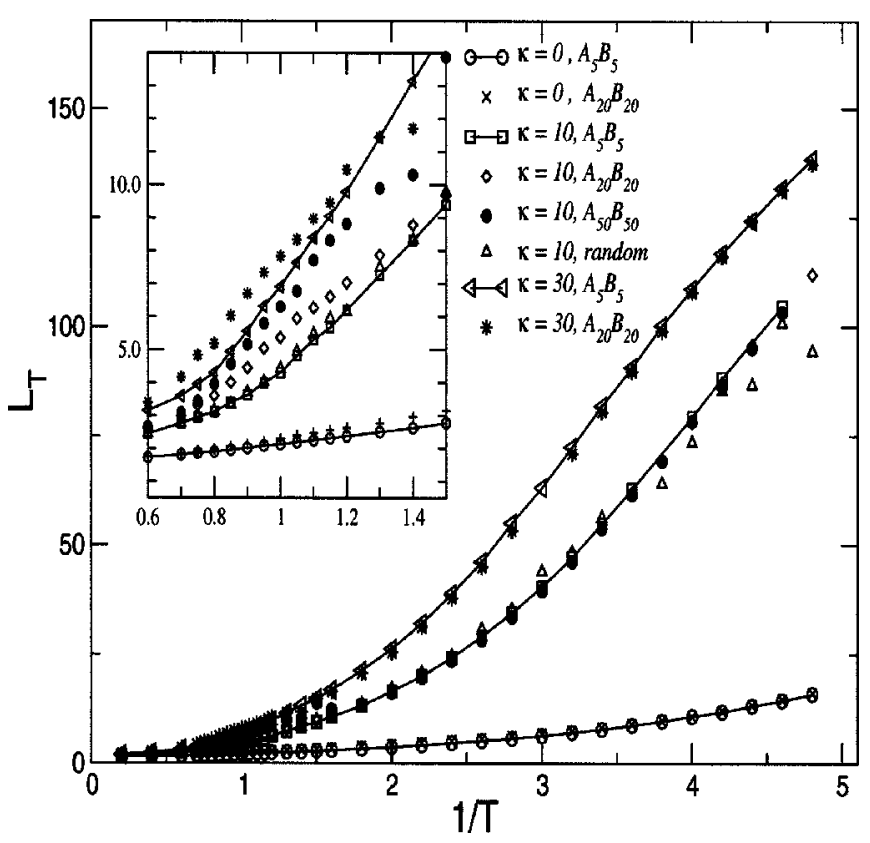

FIG. 12. Train length $L_{t}$ vs $1 / T$ for different copolymer structures and rigidities. The rest of parameters are set to $N=200, \epsilon_{A}=-1$, and $\epsilon_{B}=-0.5$. Inset plot: zoom of the deadsorbed-adsorbed transition region.

of blocks $A$ and $B$. Particularly, $T_{c A}>T_{c B}$ since $\left|\epsilon_{A}\right|>\left|\epsilon_{B}\right|$. By reducing the value of $\alpha, T_{c A}$ becomes smaller and, at the same time, $T_{c B}$ increases. For $\alpha=20$ the peak associated with $T_{c B}$ is residual, and for the $A_{5} B_{5}$ copolymer structure there is only a single peak whose location is clearly dominated by the monomers with higher adsorption energy. The adsorption becomes then a single-step process like the one observed in homopolymers. At this stage, the block structure is lost and the polymer chain could be replaced by a collection of blobs containing each $2 \alpha$ monomers with an adsorbing energy $\epsilon=\left(\epsilon_{A}+\epsilon_{B}\right) / 2$.

The characteristic adsorption temperature for the block $A, T_{c A}$, as a function of the block size $\alpha$ for different chain rigidities is plotted in the inset of Fig. 11. As expected, $T_{c A}$ increases with the chain stiffness $\kappa$ and with $\alpha$. The position of the arrows indicate the value of $T_{c A}$ for a homopolymer of the same length and rigidity, composed of $A$-type monomers only. For completeness, we have also included the results for random $A B$ copolymers (we have assigned to them, arbitrarily, $\alpha=-5$ in the inset of Fig. 11). We can conclude that random $A B$ copolymers behave similarly as $A_{1} B_{1}$ copolymers.

Another magnitudes of interest in order to characterize the behavior of block copolymers are the number and length of chain trains. A train is defined as a set of consecutive monomers in the chain, all of them being adsorbed onto the surface. The typical train length $L_{t}$ is obtained as the averaged number of monomers within a train; and the number of trains $N_{t}$ is the averaged number of trains within a chain. In Fig. 12 we have plotted $L_{t}$ vs $1 / T$ for different blockcopolymer structures and rigidities. The rest of parameters have been fixed to $N=200, \epsilon_{A}=-1$, and $\epsilon_{B}=-0.5$. As expected, a reduction in temperature increases the train length. Surprisingly, the change in $L_{t}$ is apparently barely affected by the chain structure and it seems to be dominated only by the

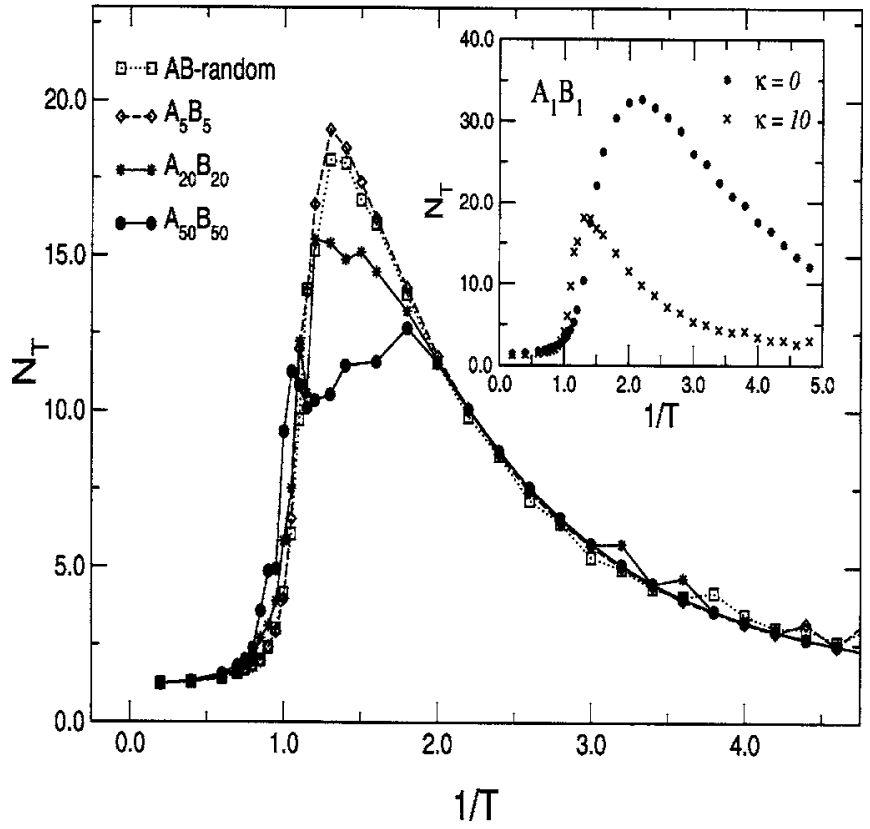

FIG. 13. Averaged number of trains $N_{t}$ vs $1 / T$ for different copolymer structures. The rest of the parameters are set to $\kappa=10, N=200, \epsilon_{A}=-1$, and $\epsilon_{B}=-0.5$. Inset: dependence of $N_{t}$ with the chain stiffness for the $A_{1} B_{1}$ structure.

stiffness. As it was pointed out by Kramarenko et al. ${ }^{23}$ in his study on homopolymer chains, a stiff chain has high disadvantage of bending, thus, the neighboring monomers of an adsorbed monomer increase their probability of being adsorbed. Therefore, we must expect that stiff chains will contain less number of trains but of major length. However, if we look in detail into what happens within the deadsorbedadsorbed transition region (see inset of Fig. 12), we realize that the chain structure does matter. The fact is that by increasing the block size $\alpha$, the length of the train, at a given temperature, is also increased.

Whereas, $L_{t}$ is a monotonous increasing function of $1 / T$, $N_{t}$ must display a completely different behavior. At high temperatures $(1 / T \rightarrow 0)$ the chain is nearly deadsorbed and $N_{t}$ $\rightarrow 0$. Below the characteristic adsorption temperature $\left(T \ll T_{c}\right)$ the chain becomes almost fully adsorbed and $N_{t}$ $\rightarrow 1$. Thus, $N_{t}$ must have a maximum value. This maximum takes place at $T=T_{c}$, as it is shown in Fig. 13. Thus, while cooling the system the number of adsorbed monomers increases generating new trains of small length. The number of trains becomes maximal at the characteristic adsorbing temperature and, a further reduction in temperature, promotes the new adsorbed monomers to link trains rather than generating new ones, thus reducing its number but increasing their length. Figure 13 also shows that the maximum number of trains at $T_{c}$ increases for copolymers with smaller block size $\alpha$. This result is expected to be even more pronounced as the difference in the adsorbing energies $\left|\epsilon_{A}-\epsilon_{B}\right|$ increases. In Fig. 13 we can observe clearly two peaks in the curve corresponding to the copolymer $A_{50} B_{50}$ in agreement with the two maxima found in the specific-heat data (Fig. 11). This result is another indication that the adsorption process takes place in two steps. The inset plot of Fig. 13 shows the behavior of $N_{t}$ for a given structure $\left(A_{1} B_{1}\right)$ but with two differ- 


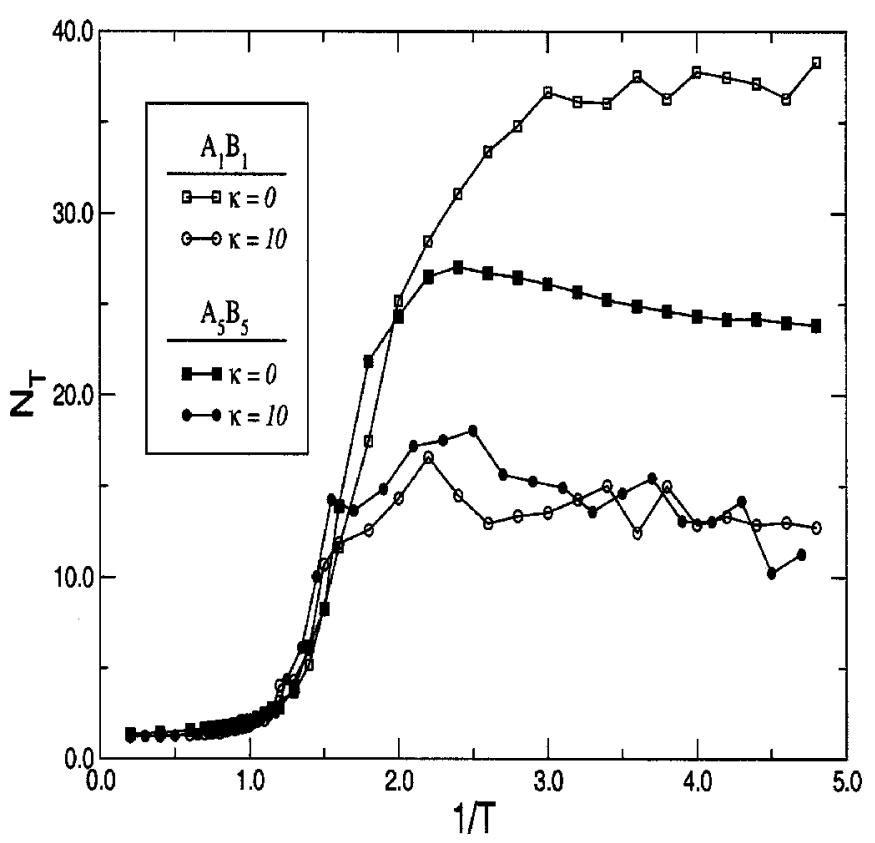

FIG. 14. $N_{t}$ vs $1 / T$ for block copolymers of small block size with the $B$-type monomers having a neutral interaction with the surface, $\epsilon_{B}=0$. The other parameters are $N=200$ and $\epsilon_{A}=-1$.

ent rigidities. The maximum for the stiffer chain shifts to higher temperatures and smaller values in agreement with the previous results. This maximum in the number of trains has been also observed in the case of flexible homopolymers. $^{14}$

If we set one of the adsorbing energies to be repulsive, for instance, $\epsilon_{B} \geqslant 0$, the polymer does not adsorb completely for a temperature small enough. In fact, $B$-type monomers are repelled from the surface and the trains are composed basically by $A$ monomers. As a result, for copolymers with a small block size, $N_{t}$ reaches a plateau value that depends on $\alpha$ and the chain stiffness $\kappa$. The results for $\epsilon_{B}=0$, and copolymer structures $A_{1} B_{1}$ and $A_{5} B_{5}$ with different rigidities, are shown in Fig. 14. This behavior is similar to one found by Zheligovskaya and Khalatur ${ }^{14}$ for flexible $A_{1} B_{1}$ copolymers.

\section{CONCLUSIONS}

In this paper we have presented the results of extensive off-lattice Monte Carlo simulations of the adsorption of semiflexible block copolymers onto flat homogeneous surfaces. The adsorption process has been analyzed under different chain lengths $(N)$, degrees of stiffness $(\kappa)$, adsorption energies for the two types of monomers $\left(\epsilon_{A}, \epsilon_{B}\right)$, and sizes of the block structure $(\alpha)$.

We have focused first in the adsorption of diblock structures. As expected, stiffer chains adsorb at a higher temperatures onto the surface, similarly to what is observed for semiflexible homopolymers. ${ }^{22}$ However, long diblock chains are found to exhibit a well-defined two-step adsorption transition, characterized by two typical adsorption temperatures that depend on the corresponding adsorption energies $T_{c}(\kappa, \epsilon)$. The critical adsorption properties have been analyzed assuming the scaling ansatz proposed by Kramarenko et al., ${ }^{23}$ originally developed for flexible homopolymers. The scaling works nicely when applied to each bock separately, but the critical exponent is no longer $\phi=0.59$. This nonuniversal behavior was already expected by Kuznetsov and Sung $^{5}$ and Gomper, ${ }^{37}$ suggesting $\phi=\phi(\kappa, \epsilon)$. The adsorption behavior of each block is barely disturbed by the presence of the other, independently of the adsorption energies. This result indicates the possibility to extend the analytical predictions of model of Whittington ${ }^{11}$ to stiff diblock chains even in the case where none of the adsorbing energies is equal to zero. The radius of gyration has been computed in the adsorbed and nonadsorbed states and we have verified that it follows the expected scaling behavior [Eq. (8)].

For stiff block-copolymer structures we have found that the separation between the two peaks in the specific-heat data, identifying the characteristic adsorption temperatures, narrows for smaller values of the block size $\alpha$. For $\alpha$ small enough we found a single peak and adsorption becomes a single-step process like in homopolymers. We have measured the length and the number of chain trains during the adsorption transition. The train length is found to increase monotonically with the block size $\alpha$ and with $1 / T$, whereas the number of trains has a maximum value (previously observed in the adsorption of flexible homopolymers ${ }^{15}$ ) located at $T=T_{c}$. We found the maximum number of trains to decrease with increasing $\alpha$. The observed effects of the chain stiffness over the chain trains may be of interest for further studies related to pattern recognition.

\section{ACKNOWLEDGMENT}

Financial support from the Spanish MEC Grant Nos. FIS2004-00953 and FIS2004-05073-C04-03 is acknowledged. KS thanks Professor Straube for encouragement and MLU, Halle for financial support.

${ }^{1}$ P. G. Khalatur, in Mathematical Methods in Contemporary Chemistry, edited by S. I. Kuchanov (Gordon and Breach, New York, 1996).

${ }^{2}$ K. De'Bell and T. Lookman, Rev. Mod. Phys. 65, 87 (1993).

${ }^{3}$ T. Vrbova and K. Prochazka, J. Phys. A 32, 5469 (1999).

${ }^{4}$ A. L. Ponomarev, T. D. Sewell, and C. J. During, Macromolecules 33, $2662(2000)$.

${ }^{5}$ D. V. Kuznestov and W. Sung, Macromolecules 31, 2679 (1998).

${ }^{6}$ K. Sumithra and K. L. Sebastian, J. Phys. Chem. 98, 9312 (1994).

${ }^{7}$ M. Muthukumar, J. Chem. Phys. 103, 4723 (1995).

${ }^{8}$ E. Orlandini, F. Seno, and A. L. Stella, Phys. Rev. Lett. 84, 294 (2000).

${ }^{9}$ Y. Singh, S. Kumar, and D. Giri, J. Phys. A 32, L407 (1999).

${ }^{10}$ C. M. Marques, J. F. Joanny, and L. Leibler, Macromolecules 21, 1051 (1998).

${ }^{11}$ S. G. Whittington, J. Phys. A 31, 3769 (1998).

${ }^{12}$ M. S. Moghaddam, T. Vrbova, and S. G. Whittington, J. Phys. A 33, 4573 (2000).

${ }^{13}$ Th. C. Clancy and S. E. Webber, Macromolecules 26, 628 (1993).

${ }^{14}$ E. A. Zheligovskaya and P. G. Khalatur, J. Chem. Phys. 106, 8598 (1997).

${ }^{15}$ E. A. Zheligovskaya, P. G. Khalatur, and A. R. Khokhlov, Phys. Rev. E 59, 3071 (1999).

${ }^{16}$ A. R. Khokhlov and P. G. Khalatur, Phys. Rev. Lett. 82, 3456 (1999).

${ }^{17}$ S. Srebnik, A. K. Chackraborty, and E. I. Shakhnovich, Phys. Rev. Lett. 77, 3157 (1996).

${ }^{18}$ K. Sumithra and A. Baumgärtner, J. Chem. Phys. 109, 1540 (1998).

${ }^{19}$ J. S. Shaffer, Macromolecules 27, 2987 (1994).

${ }^{20}$ O. Kratky and G. Porod, Rec. Trav. Shim 68, 1106 (1949).

${ }^{21}$ Z. Y. Chen and S. M. Cui, Phys. Rev. E 52, 3876 (1995).

${ }^{22}$ T. Sintes, K. Sumithra, and E. Stranbe, Macromolecules 34, 1352 (2001).

${ }^{23}$ E. Y. Kramarenko, R. G. Winkler, and P. G. Khalatur, J. Chem. Phys. 
104, 4806 (1996).

${ }^{24}$ C. C. Van der Linden, F. A. M. Leermakers, and G. J. Fleer, Macromolecules 29, 1000 (1996); 29, 1172 (1996).

${ }^{25}$ J. F. Joanny and A. Johner, J. Phys. II (France) 6, 511 (1996).

${ }^{26}$ G. L. Kenausis, J. Volrols, D. L. Elbert, N. Huang, R. Hofer, L. R. Taylor, M. Textor, J. A. Hubbell, and N. D. Spencer, J. Phys. Chem. B 104, 3298 (2000).

${ }^{27}$ N.-P. Huang, R. Michel, J. Voros, M. Textor, R. Hofer, A. Rossi, D. L. Elbert, J. A. Hubbell, and N. D. Spencer, Langmuir 17, 489 (2001).

${ }^{28}$ R. J. Chen, S. Bangsaruntip, A. Drouvalakis, N. Wong Shi Kam, M. Shim, Y. Li, W. Kim, P. J. Utz, and H. Dai, PNAS 100, 4984 (2003).

${ }^{29}$ A. S. Doherty, K. D. Berglund, A. Buchholz, I. V. Kourkine, T. M. Przybycien, R. D. Tilton, and A. E. Barron, Electrophoresis 23, 2766 (2002).
${ }^{30}$ D. C. Schwartz and A. Samad, Curr. Opin. Biotechnol. 8, 70 (1997).

${ }^{31}$ W. N. Wang, J. Y. Lin, and D. C. Schwartz, Biophys. J. 75, 513 (1998).

${ }^{32}$ A. Baumgärtner, in Applications of the Monte Carlo Method in Statistical Physics, Topics in Current Physics Vol. 36, 2nd. ed., edited by K. Binder (Springer-Verlag, Berlin, 1987).

${ }^{33}$ T. Xiang, Biophys. J. 65, 1108 (1993).

${ }^{34}$ M. Allen and D. Tildesley, Computer Simulation of Liquids (Clarendon, Oxford, 1987)

${ }^{35}$ E. Eisenriegler, K. Kremer, and K. Binder, J. Chem. Phys. 77, 6296 (1982).

${ }^{36}$ P.-G. de Gennes and P. Pincus, J. Phys. (France) Lett. 44, L-241 (1983).

${ }^{37}$ G. Gompper, in Biologically Inspired Physics, edited by L. Peliti (Plenum, New York, 1991). 